\title{
Synthesis and biological activity of some new 4-thiazolidinone derivatives
}

\author{
V. P. Trivedi*, N. K. Undavia and P. B. Trivedi \\ University Department of Chemistry, Bhavnagar University, Bhavnagar-364 002, India \\ i. \\ Manuscript received 16 April 2003, revised 15 October 2003, accepted 12 January 2004
}

\begin{abstract}
4-Thiazolidinone is a medicinally important moiety. We have used thiourea with other potential moiety 4 quinazolinone to enhance medicinal value of the 4-thiazolidinones moiety. 15 new compounds are reported along with their spectral data, antibacterial and antitubercular activities.
\end{abstract}

4-Thiazolidinones have been found to be endowed with a variety of biological activities ${ }^{1-5}$. Keeping in view the medicinal importance and utility of thiazolidine moiety, it was worth attempting to synthesize some new potential 4oxothiazolidines in which the side chain of actithiazic acid in position-2 is shifted to position-5 in 4-thiazolidinone with substituting other groups in the ring. In the present paper, we have incorporated well-known organic synthon thiourea with another potential moiety 4-quinazolinone to augment medicinal value of the compounds. All the new compounds were screened for their antibacterial and antitubercular activities.

\section{Results and discussion}

\section{Biological activity :}

The compounds were screened for antibacterial activity using cup-plate method ${ }^{6}$. The testing was carried out in DMF solution at a concentration of $50 \mu \mathrm{g}$ $\mathrm{ml}^{-1}$ using gram positive bacteria like Staphylococcus aureus and gram negative bacteria like Escherichia coli and Salmonella typhosa. Chloramphenicol was used as a standard drug.

Antitubercular activity of the synthesized compounds :

This activity was carried out at TAACF (Tuberculosis Antimicrobial Acquisition and Coordinating Facility), Southern Research Institute, Birmingham, Alabama. The following procedure was used. The TAACF integrates the various components of the drug discovery and development process into a unified program that facilitates the search for antimycobacterial drugs. This program consists of four closely interwoven components; a compound acquisition, repository, and data analysis service operated by Southern Research Institute; an in vitro screening service at the GWL Hansen's Disease Center; and in vivo screening service at Colorado State University; and a technology transfer service at Research Triangle Institute.
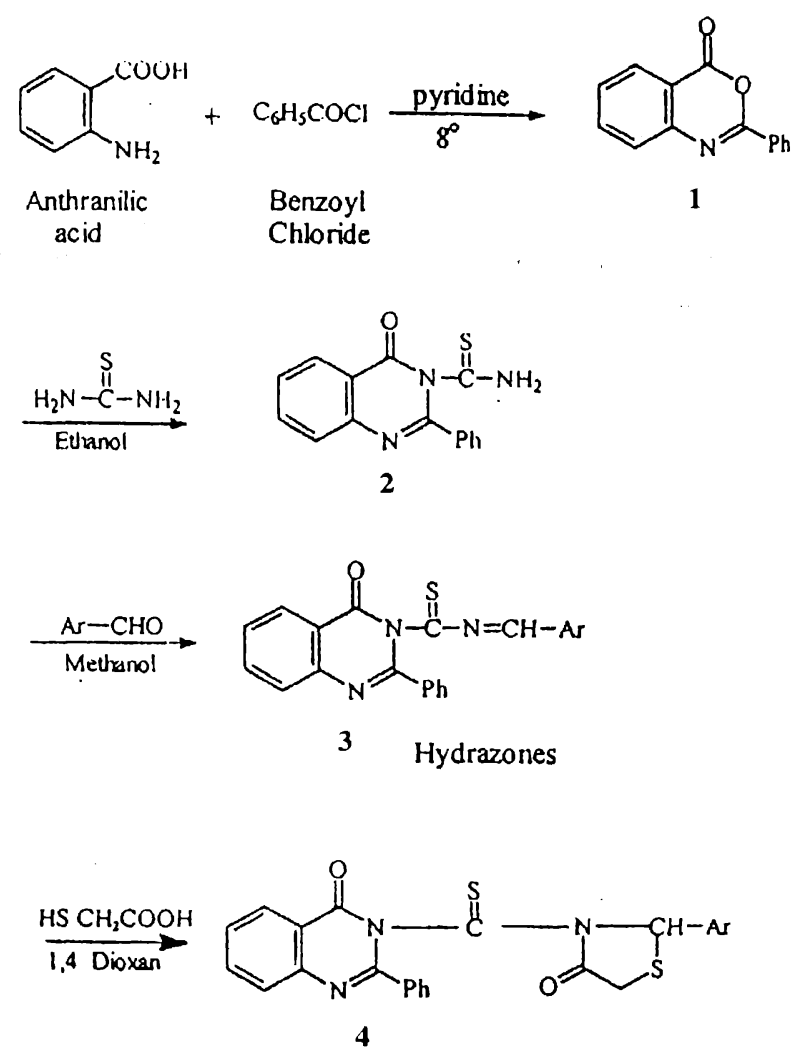

where, $\mathrm{Ar}=$ different aryl groups cited in Table 1.

Under the direction of the U.S. National Institute of AIlergy and Infectious Diseases (NIAID), Southern Research Institute coordinates the overall program. All services are provided confidentially and at no cost to the supplier.

In vitro evaluation of antimycobacterial activity :

Primary screening conducted at $12.50 \mathrm{\mu g} \mathrm{m}^{-1}$ (or molar equivalent of highest molecular weight compound in a series of congeners) against Mycobacterium tuberculosis $\mathrm{H}_{37} \mathrm{R}_{\mathrm{v}}$ (ATCC 27294) in BACTEC 12B medium using a broth microdilution assay, the Microplate Alamar 


\begin{tabular}{|c|c|c|c|c|c|}
\hline \multicolumn{6}{|c|}{ Table 1. Physical data for the synthesized comounds* } \\
\hline Compd. & Sample & $\mathrm{Ar}$ & Molecular & Yield & M.p. \\
\hline & ID & & formula & $\%$ & ${ }^{\mathrm{C}} \mathrm{C}$ \\
\hline $4 a$ & 125312 & $\mathrm{C}_{6} \mathrm{H}_{5}$ & $\mathrm{C}_{24} \mathrm{H}_{17} \mathrm{~N}_{3} \mathrm{O}_{2} \mathrm{~S}_{2}$ & 71.20 & 121 \\
\hline b & 125313 & $2(\mathrm{OH}) \mathrm{C}_{6} \mathrm{H}_{4}$ & $\mathrm{C}_{24} \mathrm{H}_{17} \mathrm{~N}_{3} \mathrm{O}_{3} \mathrm{~S}_{2}$ & 67.00 & 137 \\
\hline c & 125314 & $3(\mathrm{OH}) \mathrm{C}_{6} \mathrm{H}_{4}$ & $\mathrm{C}_{24} \mathrm{H}_{17} \mathrm{~N}_{3} \mathrm{O}_{3} \mathrm{~S}_{2}$ & 69.40 & 183 \\
\hline d & 125315 & $4(\mathrm{OH}) \mathrm{C}_{6} \mathrm{H}_{4}$ & $\mathrm{C}_{24} \mathrm{H}_{17} \mathrm{~N}_{3} \mathrm{O}_{3} \mathrm{~S}_{2}$ & 70.30 & 133 \\
\hline e & 125316 & $4(\mathrm{Cl}) \mathrm{C}_{6} \mathrm{H}_{4}$ & $\mathrm{C}_{24} \mathrm{H}_{16} \mathrm{~N}_{3} \mathrm{O}_{2} \mathrm{~S}_{2} \mathrm{Cl}$ & 68.00 & 175 \\
\hline f & 125317 & $4\left(\mathrm{OCH}_{3}\right) \mathrm{C}_{6} \mathrm{H}_{4}$ & $\mathrm{C}_{25} \mathrm{H}_{19} \mathrm{~N}_{3} \mathrm{O}_{3} \mathrm{~S}_{2}$ & 71.60 & 104 \\
\hline g & 125318 & $3,4,5\left(\mathrm{OCH}_{3}\right)_{3} \mathrm{C}_{6} \mathrm{H}_{2}$ & $\mathrm{C}_{27} \mathrm{H}_{23} \mathrm{~N}_{3} \mathrm{O}_{5} \mathrm{~S}_{2}$ & 69.00 & 111 \\
\hline h & 125319 & $2(\mathrm{Cl}) \mathrm{C}_{6} \mathrm{H}_{4}$ & $\mathrm{C}_{24} \mathrm{H}_{16} \mathrm{~N}_{3} \mathrm{O}_{2} \mathrm{~S}_{2} \mathrm{Cl}$ & 67.80 & 163 \\
\hline $\mathbf{i}$ & 125320 & $3,4(\mathrm{Cl})_{2} \mathrm{C}_{6} \mathrm{H}_{3}$ & $\mathrm{C}_{24} \mathrm{H}_{15} \mathrm{~N}_{3} \mathrm{O}_{2} \mathrm{~S}_{2} \mathrm{Cl}_{2}$ & 70.00 & 125 \\
\hline $\mathbf{j}$ & 125321 & $3\left(\mathrm{NO}_{2}\right) \mathrm{C}_{6} \mathrm{H}_{4}$ & $\mathrm{C}_{24} \mathrm{H}_{16} \mathrm{~N}_{4} \mathrm{O}_{4} \mathrm{~S}_{2}$ & 65.00 & 97 \\
\hline k & 125322 & $4\left(\mathrm{CH}_{3}\right) \mathrm{C}_{6} \mathrm{H}_{4}$ & $\mathrm{C}_{25} \mathrm{H}_{19} \mathrm{~N}_{3} \mathrm{O}_{2} \mathrm{~S}_{2}$ & 65.90 & 119 \\
\hline 1 & 125323 & $-\mathrm{CH}=\mathrm{CH}-\mathrm{C}_{6} \mathrm{H}_{5}$ & $\mathrm{C}_{26} \mathrm{H}_{19} \mathrm{~N}_{3} \mathrm{O}_{2} \mathrm{~S}_{2}$ & 71.00 & 91 \\
\hline $\mathbf{m}$ & 125324 & $3,4-\mathrm{O}-\mathrm{CH}_{2}-\mathrm{O}-\mathrm{C}_{6} \mathrm{H}_{3}$ & $\mathrm{C}_{25} \mathrm{H}_{17} \mathrm{~N}_{3} \mathrm{O}_{4} \mathrm{~S}_{2}$ & 69.40 & 157 \\
\hline $\mathrm{n}$ & 125325 & $3\left(\mathrm{OCH}_{3}\right) 4(\mathrm{OH}) \mathrm{C}_{6} \mathrm{H}_{3}$ & $\mathrm{C}_{25} \mathrm{H}_{19} \mathrm{~N}_{3} \mathrm{O}_{4} \mathrm{~S}_{2}$ & 70.50 & 115 \\
\hline $\mathbf{o}$ & 125326 & $2(\mathrm{OH}) 5(\mathrm{Br}) \mathrm{C}_{6} \mathrm{H}_{3}$ & $\mathrm{C}_{24} \mathrm{H}_{16} \mathrm{~N}_{3} \mathrm{O}_{3} \mathrm{~S}_{2} \mathrm{Br}$ & 73.70 & 178 \\
\hline
\end{tabular}

Blue Assay (MABA) ${ }^{7}$. Compounds exhibiting fluorescence are tested in the BACTEC 460 radiometric system. Compounds effecting $<90 \%$ inhibition in the primary screen (i.e., MIC $>12.50 \mu \mathrm{g} \mathrm{ml}^{-1}$ ) are not generally evaluated further.

Minimum compound requirements : $1.0 \mathrm{mg}$.

\section{Conclusion :}

Most of the compounds were found moderately active (14-26 $\mathrm{mm}$ zone of inhibition). Comparatively significant activity was observed in compound bearing substituents 2-hydroxy-5-bromophenyl (24), 4-hydroxyphenyl (20), 2-chlorophenyl (22), 4-chlorophenyl (24), 4-methoxyphenyl (26) and 3-nitrophenyl (20 mm). None of the synthesized compounds was found active at 12.50 $\mu \mathrm{g} \mathrm{ml}^{-1}$ concentration against Mycobacterium tuberculosis $\mathrm{H}_{37} \mathrm{R}_{\mathrm{v}}$.

\section{Experimental}

All melting points were taken in open capillaries and are uncorrected. The structures of the new products were confirmed by the elemental and spectral analysis. IR spectra $(\mathrm{KBr})$ were recorded on a Shimadzu FTIR spectrophotometer, ${ }^{1} \mathrm{H}$ NMR spectra $\left(\mathrm{CDCl}_{3}+\mathrm{TFA}\right)$ on Bruker $(200 \mathrm{MHz})$ and Hitachi $1200(60 \mathrm{MHz})$ spectrometers using TMS as internal reference and mass spectra on a Jeol JMSD-300 spectrometer.

Synthesis of 2-phenyl-3-1-benzoxazin-4(4H)-one ${ }^{8}$ (1) : Benzoyl chloride (140.5 g; $1 M$ ) was added dropwise to anthranilic acid $(137 \mathrm{~g} ; 1 \mathrm{M})$ dissolved in pyridine $(60$ $\mathrm{ml}$ ) with constant stirring at $8^{\circ}$ over the period of $1 \mathrm{~h}$. After the completion of addition, the reaction mixture was stirred for half an hour at RT. At the end of the reaction, solid mass was obtained. It was filtered, washed successively with sodium bicarbonate solution (to remove unreacted acid) and then water, dried and recrystallised from rectified spirit. Yield (178.5 g, 80\%), m.p. $114^{\circ}$ (Found : $\mathrm{N}, 6.13, \mathrm{C}_{14} \mathrm{H}_{9} \mathrm{NO}_{2}$ requires : $\mathrm{N}, 6.27 \%$ ).

Synthesis of 2-phenyl-3-thiocarboxamide-4-oxoquinazoline $^{8}(2)$ :

2-Phenyl-3,1-benzoxazin-4(4H)-one (111.5 g; $0.5 \mathrm{M})$ was treated with equimolar proportion of thiourea $(38 \mathrm{~g}$; $0.5 \mathrm{M})$ using ethanol $(250 \mathrm{ml})$ as solvent. The contents were refluxed for $3 \mathrm{~h}$. The excess of solvent was then distilled off, and the resulting solid was dried and recrystallised from rectified spirit. Yield $(101.10 \mathrm{~g}, 72 \%)$, m.p. $189^{\circ}$ (Found : N, 14.87; S, 11.25. $\mathrm{C}_{15} \mathrm{H}_{11} \mathrm{~N}_{3} \mathrm{OS}$ requires $\mathrm{N}, 14.93 ; \mathrm{S}, 11.38 \%)$.

Synthesis of 2-phenyl-3-(2-hydroxybenzaliminothiocarbonyl)-4-oxoquinazoline ${ }^{9}(3)$ :

A mixture of 2-phenyl-3-thiocarboxamido-4-oxoquinazoline $(2.81 \mathrm{~g} ; 0.01 \mathrm{M})$ and salicylaldehyde $(1.22 \mathrm{~g}$; $0.01 M)$ in methanol was refluxed on a water-bath for 3 h. The clear solution was poured in ice-water, filtered the solid and recrystallised from ethanol $(95 \%)$. Yield (3.11 g, 81\%), m.p. $207^{\circ}$ (Found : N, 10.85; S, 8.22. $\mathrm{C}_{22} \mathrm{H}_{15} \mathrm{~N}_{3} \mathrm{O}_{2} \mathrm{~S}$ requires : N, 10.90; $\mathrm{S}, 8.31 \%$ ); $v_{\max } 3065$ 
(-CH str., aromatic), 1660 ( $>\mathrm{C}=\mathrm{O}$ str., quinazoline), 3415 (-OH phenolic), $1650(-\mathrm{CH}=\mathrm{N}-), 1259$ (>C=S str.), 1545 $\mathrm{cm}^{-1}(\mathrm{C}=\mathrm{C}$ str., quinazoline $)$ and ${ }^{2} \mathrm{H}$ NMR $\delta 6.8(1 \mathrm{H}, \mathrm{s}$, $-\mathrm{CH}=), 7.6-8.2(\mathrm{~m}, \mathrm{Ar}-\mathrm{H}), 8.5(\mathrm{~s}, \mathrm{Ar}-\mathrm{OH})$.

Synthesis of 2-(2-hydroxyphenyl)-3-[(2-phenyl-4-oxo3-quinazolinyl)-thiocarbonyl]-4-oxo-thiazolidine (4) :

To a solution of 2-phenyl-3-(2-hydroxybenzaliminothiocarbonyl)-4-oxo-quinazoline ( $1.92 \mathrm{~g} ; 0.005 M)$ in 1,4dicxan $(25 \mathrm{ml})$ was added thioglycolic acid $(0.464 \mathrm{~g} ; 0.005$ $M)$ with stirring. The mixture was refluxed on steambath for $8 \mathrm{~h}$, cooled and poured into socium bicarbonate solution $(4 N)$. The resulting solid was washed with water, dried and recrystallised from rectified spirit. Yield ( 1.53 g, 67\%), m.p. $137^{\circ}$ (Found : N, 9.09; S, 13.75. $\mathrm{C}_{24} \mathrm{H}_{17} \mathrm{~N}_{3} \mathrm{O}_{2} \mathrm{~S}_{2}$ requires : $\mathrm{N}, 9.15 ; \mathrm{S}, 13.94 \%$ ).

Other compounds reported in Table 1 were synthesized following the same procedure.

Spectral data of the synthesized compound (4) :

$v_{\max } 3065$ (-CH str., aromatic), 1764.5 (C=O str., thiazolidine), 1660 (C=O str., quinazoline), $1620(\mathrm{C}=\mathrm{N}$ str., quinazoline), 1572 (C-N str., thiazolidine), $1525(\mathrm{C}=\mathrm{C}$ str., quinazoline), 685 (C-S-C str., thiazolidine), 1240 (>C=S str.,) 3415 (-OH phenolic), $1433 \mathrm{~cm}^{-1}(\mathrm{CH}$ deformation of $-\mathrm{CH}_{2} \mathrm{CO}$ - group); $\delta 3.5\left(2 \mathrm{H}\right.$, singlet, $\left.\mathrm{CH}_{2} \mathrm{~S}\right)$, 5.35 ( $1 \mathrm{H}$, singlet, $\mathrm{CH}-\mathrm{Ar}$ ), 7.6-8.2 (multiplate, $\mathrm{Ar}-\mathrm{H}$ ), 10.5 $(-\mathrm{OH}$, singlet $), 6.14(1 \mathrm{H}$, thiazolidine $)$.

\section{Acknowledgement}

The authors are thankful to the authorities of Bhavnagar University for facilities and Dr. Cecil D. Kwong, TAACF Medicinal Chemist, Southern Research Institute, U.S.A. for antitubercular assay and Dr. H. H. Patel, Life Sciences Department for biological screening.

\section{References}

1. P. C. Eisman, E. A. Kanopka and R. L. Mayer, Am. Rev. Tubercul. Pulmonary Diseases, 1954, 70, 121; 1958, 77, 694; R. R. Shah, R. D. Mehta and A. R. Parikh, J. Inclian Chem. Soc., 1981, 58 113; K. Venkatesh, Ph.D. Thesis, Bombay University, 1979; J. M. Turel, Ph.D. Thesis, South Gujarat University, 1977; K. N. Bhatt. Ph.D. Thesis, Bhavnagar University, 1987.

2. A. J. Srivastava, S. Swaroop, V. K. Saxena, B. L. Chowdhari and P. Srivastava, Indian J. Pharm. Sci., 1982, 51.

3. A. R. Bhatt, S. Sankarasubramanian and K. T. Jose, Indian Drugs, 1983, 20, 383.

4. A. K. Elshafei and K. M. Hasson, Curr. Sci., 1983, 52, 633.

5. E. Rajanarendar, Md. Afzal and D. Karunakar, Indian J. Chem., Sect. B, 2003, 42, 353.

6. F. Cavanagh, "Analytical Microbiology", Academic, New York. 1963, p. 126.

7. L. Collins and S. G. Franzblau, Antimicrob. Agents Chemother, 1997, 41. 1004.

8. M. B. Deshmukh and D. S. Deshmukh, J. Indian Chem. Soc., 1995, 72, 847

9. Zaunschirm, Ann., 1888, 245, 279. 